\title{
Study on Evaluation Model of Attribute Barycentric Coordinates
}

\author{
Guanglin $\mathrm{Xu}$ and Xiaolin $\mathrm{Xu}^{*}$ \\ College of Mathematics and Information, Shanghai Lixin University of \\ Commerce,Shanghai, China \\ College of International Vocational Education,Shanghai Second Polytechnic \\ University, Shanghai, China \\ glxu@outlook.com,xlxu2001@163.com
}

\begin{abstract}
The core of the comprehensive evaluation problem is how to distribute the weight of each attribute. The attribute coordinate evaluation model (ACEM) has realized dynamic distribution for weight according to the evaluator preference curve, but there still is a problem that maybe a few evaluation results are unreasonable after transforming from locally satisfactory degree to globally satisfactory degree. In order to solve this problem, this paper proposes the improved attribute coordinate evaluation model (IACEM) which directly calculates the global satisfaction, simultaneously, gives the principle to determine whether the final evaluation result is reasonable or not, namely, the principle of weight priority is adopted for the evaluated objects with the same total score level, and the principle of total score priority is adopted for the evaluated objects with different total score levels. To eliminate the errors caused by the change of total score planes, this paper also proposes a parameter adjustment method. The final simulation verifies the correctness and the rationality of the improved model.
\end{abstract}

Keywords: Comprehensive evaluation; Dynamic weight; Attribute Barycentric Coordinates

\section{Introduction}

The core of comprehensive evaluation problem is how to allocate the weight of each index, which has been studied by the scholars from different angles. T. L. Saaty proposed AHP in references [1, 2], and further proposed ANP method that structures decision problem as a network $[3,4]$. The fuzzy pair wise comparison is used to allocate weight for each index in reference [5]. The benefit of ACEM is to dynamically allocate the weight according to the preference curve of the evaluator. At present, whether the theory or practice has obtained some achievement [6-15], but also encounter some problems. For example, during the transforming from the local satisfaction degree to the global satisfaction degree, there exist a small number of unreasonable results, however; there is no principle and standard to evaluate if the result getting by ACEM is reasonable or not. In order to avoid errors from transforming from the local to the global, this paper tries to improve ACEM by calculating satisfaction solution directly from a global point of view, moreover, and gives a principle to validate if the result produced by ACEM is reasonable or not, and the method of adjustment on the evaluation is given. In this paper, the first part introduces the basic theory of ACEM, the second part analyzes the problems which have been encountered, the third part improves the model and puts forward the model

\footnotetext{
* Corresponding Author. The two authors contributed to this work.

This work was supported by Phase-4, Software Engineering (Service Engineering) under Grant No. XXKZD1301.
} 
validation principle and the method for adjustment on evaluation results, and the last part carries out the simulation and experimental validation.

\section{Theory of ACEM}

For multi-attribute comprehensive evaluation problem, if all the attributes are endowed with uniform dimension, all attributes of the best idea scheme should have full mark (assuming that full mark is 10 points). However, in reality there is no principle of optimality, so the principle of satisfaction is generally adopted, in other words, what we get is only the satisfactory solution, not the optimal solution. Thus, evaluation result can only be the most satisfactory solution that meets certain weight conditions. The most satisfactory solution can be expressed as the extremum problem of the following utility function $\mathrm{u}$.

$$
\operatorname{Max}_{i}\left\{\mathrm{u}=\sum_{j=1}^{m} w_{j} x_{i j}\right\}
$$

Where $0 \leq x_{i j} \leq 10$ is the value of the $i_{\text {th }}$ evaluated object with respect to the $j_{\text {th }}$ decision attribute $a_{j} . \bar{W}=\left(w_{1}, \cdots, w_{m}\right)$ is the weight (or preference) of the evaluator with respect to attribute vector,, and meets $\sum_{j=1}^{m} w_{j}=1$ the solution of the problem (1) exists, it is very difficult to find it among the whole utility value space $U=\left\{u=\sum_{j=1}^{m} w_{j} x_{i j}\right\}$.

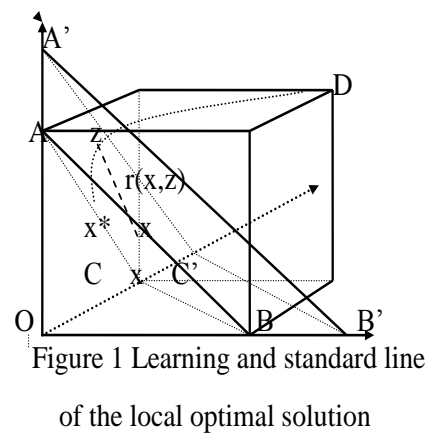

ACEM uses the following procedures to find the solution of problem (1): Firstly divides the solution of problem (1) into a series of sub-problems to find most satisfactory local solutions; then, find the most satisfactory global solution in the collection of all the local satisfactory solutions. The following shows how to get a special most satisfactory local solution.

Let $X=\left\{x_{i}=\left(x_{i 1}, \ldots, x_{i m}\right) \mid x_{i j}\left(0 \leq x_{i j} \leq 10\right)\right\}$ be all evaluated objects space (under threedimension, $X$ is the cube in Figure 1$), S_{10}=\left\{x_{i}=\left(x_{11}, \ldots, x_{i m}\right) \mid=10\right\}$, then $S_{10} \cap X$, their intersection will constituted a $(\mathrm{m}-1)$-dimension simplex $(\triangle \mathrm{ABC}$ in Figure 1) with equal total score. Because the mathematical meaning of $\vec{W}=\left(\mathrm{w}_{1}, \ldots, \mathrm{w}_{\mathrm{m}}\right)$ means that a whole weight is divided into several parts and assigned to each attributes. Therefore, in simplex $\mathrm{S}_{10} \cap \mathrm{X}$ with equal total score ( $=10$ points), total 10 score will be distributed into the object with $\mathrm{m}$ attributes: $x^{*}=\left(x_{1}^{*}, \ldots x_{m}^{*}\right)=10 \times \vec{W}=\left(10 \times \mathrm{w}_{1}, \ldots, 10 \times \mathrm{w}_{\mathrm{m}}\right)$. Besides, 
$\vec{W}=\left(\mathrm{w}_{1}, \ldots, \mathrm{w}_{\mathrm{m}}\right)$ will be exactly barycentric coordinates of $x^{*}$ in the simplex $\mathrm{S}_{10} \cap \mathrm{X}$, namely, $x^{*}=\left(\mathrm{w}_{1}, \ldots, \mathrm{w}_{\mathrm{m}}\right), \sum_{j=1}^{m} w_{j}=1$ . Its physical significance can be explained as: if place each weight component $\mathrm{w}_{\mathrm{j}, \mathrm{j}}=1, \ldots \mathrm{m}$ on the vertex $\mathrm{a}_{\mathrm{j},}$ of $\mathrm{S}_{10} \cap \mathrm{X}$, then $x^{*}$ will be exactly the physical barycentric point. Therefore, no matter from the aspects such as mathematical meaning of weight, physics, algebraic topology or linear space, etc., $x^{*}=\left(10 \times \mathrm{w}_{1}, \ldots, 10 \times \mathrm{w}_{\mathrm{m}}\right)$ should be the most satisfactory solution distributed by $\vec{W}=\left(\mathrm{w}_{1}, \ldots, \mathrm{w}_{\mathrm{m}}\right)$ in $\mathrm{S}_{10} \cap \mathrm{X}$.

Obviously, if there is $x^{*}$ in $S_{10} \cap X$, the most satisfactory local solution will be confirmed. Otherwise, if $x^{*}$ doesn't exist, the distance between any evaluated object $X_{i}=\left(x_{i 1}, \cdots, x_{i m}\right)$ and $x^{*}$ is able to express the distance between the evaluated object $x$ and the most satisfactory local solution $x^{*}$ of the evaluator $z$. The satisfaction function of $z$ is assumed as follows.

$\operatorname{sat}\left(x_{i}, z\right)=\lambda\left(x_{i}, x^{*}(z)\right) g\left(r\left(x_{i}, x^{*}(z)\right)\right)$

Where $\lambda\left(x_{i}, x^{*}(z)\right)$ is a global satisfaction equation. For discussing simply, temporarily, let $\lambda\left(x_{i}, x^{*}(z)\right)=1 . g\left(r\left(x_{i}, x^{*}(z)\right)\right)$ is an equation to reflect the similarity between $x_{i}$ and standard point $x^{*}$. Considering that the $g\left(r\left(x_{i}, x^{*}(z)\right)\right)$ meets the characteristic: when $r\left(x, x^{*}(z)\right)=0$, then $g\left(r\left(x_{i}, x^{*}(z)\right)\right)=1$, when $r\left(x, x^{*}(z)\right)=\infty, g\left(r\left(x_{i}, x^{*}(z)\right)\right)=0$, and the above obtained the sum of qualitative mapping function exactly has these characteristics, thus let $\operatorname{sat}\left(x_{i}, x^{*}(\mathrm{z})\right)$ as:

$$
\operatorname{sat}\left(x_{i}, x^{*}(\mathrm{z})\right)=\exp \left(-\frac{\sum_{j=1}^{m} w_{j}\left|x_{i j}-x_{j}^{*}(z)\right|}{\sum_{j=1}^{m} \delta_{j}\left(x_{j}^{*}(z)-\delta_{j}\right)}\right)
$$

Where $\delta_{j}=\delta_{j}(z)$ is the error of the evaluator $z$ with respect to the $j_{\text {th }}$ attribute, the weight $w_{j}=\mathrm{w}_{\mathrm{j}}\left(\mathrm{x}_{\mathrm{j}}^{*}(\mathrm{z}), \delta_{\mathrm{j}}\right)$ is the function of $x_{j}^{*}(z)$ and $\delta_{i}$.The equation (3) shows satisfaction of the evaluator $z$, so (3) is called the local satisfaction function of the evaluator on scheme in $S_{10} \cap X \quad X_{i}=\left(10 \times w_{1}, \cdots, 10 \times w_{m}\right)$ should be accordant with most satisfactory local solution distributed by weight $\vec{W}=\left(\mathrm{w}_{1}, \cdots, \cdot \mathrm{w}_{\mathrm{m}}\right)$, then according to (4) 


$$
x * \mid \mathrm{T}=\mathrm{T} \times \bar{W}=\left(\mathrm{T} \times \mathrm{w}_{1}, \cdots, \mathrm{T} \times \mathrm{w}_{\mathrm{m}}\right)
$$

$$
\text { Where } \sum_{j=1}^{m} x_{i j}=T \succcurlyeq 10 \text {. is the obtained scheme }{ }^{x^{*} \mid \mathrm{T}} \text { the most satisfactory local }
$$

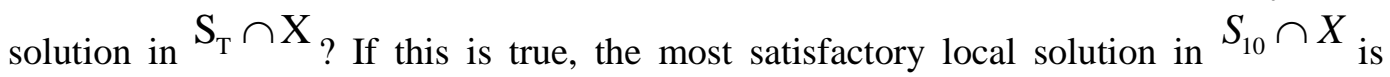
solved by equation ( 3 ) for all the $\mathrm{T}$, then the most satisfactory global solution is able to be found in the most satisfactory local solutions set $\left\{x^{*}=\left(x_{1}^{*}, \cdots, x_{m}^{*}\right) \mid \mathrm{T}, 10 \leq \mathrm{T} \leq 10 \times \mathrm{n}\right\}$.

However, the absolute satisfactory solution $D=\left(10_{1}, \cdots 10_{m}\right)\left(\neq T \times \overline{\left.W=T \times\left(w_{1} \cdots \cdots w_{w}\right)\right)}\right.$, unless $T=10 \times m$, and the fact that $w_{j}=1 / m,(j=1, \cdots, m)$ is irrelevant to the psychological weight of the evaluator $\vec{W}$ shows when total score $T \geq 10$, the most satisfactory solution $\left\{x^{*}=\left(x_{1}^{*}, \cdots, x_{m}^{*}\right) \mid \mathrm{T}_{\text {in }} S_{T} \cap X\right.$ can't be simply obtained according to equation (3). If

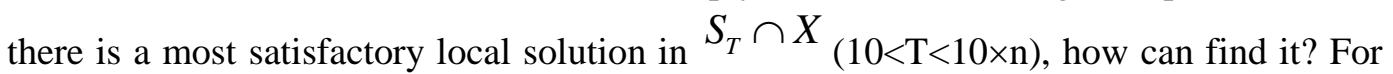
this, ACEM uses the machine learning method to find and gradually approach the method for the most satisfactory local solution $\left\{x^{*}=\left(x_{1}^{*}, \cdots, x_{m}^{*}\right) \mid \mathrm{T}\right.$, and then the global satisfactory function is used to calculate satisfaction conversion from local to the global.

\subsection{The Most Satisfactory Local Solution under Certain Constraint Conditions}

$$
\mathrm{S}_{\mathrm{T}}=\left\{\mathrm{x}_{\mathrm{i}}=\left(\mathrm{x}_{\mathrm{i} 1}, \cdots, \mathrm{x}_{\mathrm{im}}\right) \mid \sum_{\mathrm{j}=1}^{\mathrm{m}} x_{i j}=\mathrm{T}\right\}
$$

Let

be the hyperplane whose total score equal $\mathrm{T}$, then the intersection between $\mathrm{S}_{\mathrm{T}}$ and $\mathrm{X}$ is a (n-1)-dimension simplex. (shown in Figure $1, S_{T} \cap X=\triangle \mathrm{ABC}, \Delta \mathrm{A}^{\prime} \mathrm{B}^{\prime} \mathrm{C}^{\prime}$ is the two-dimension simplex that is obtained by the way that the equal total score hyperplane $\mathrm{S}_{\mathrm{T}}$ sections $\left.\mathrm{X}, S_{T} \cap X=\Delta \mathrm{A}^{\prime} \mathrm{B}^{\prime} \mathrm{C}^{\prime}\right)$. Let $\left\{x_{k}, k=1, \cdots, s\right\} \subseteq S_{T} \cap X$ be the set of sample scheme ${ }^{x_{i}}$ whose total score equals T. If the evaluator $\mathrm{z}$ selects $\mathrm{t}$ sets of relatively satisfactory schemes $\left\{x_{h}, k=1, \cdots, s\right\}$ from $\left\{x_{\mathrm{k}}\right\}$, and marks as $v_{h}\left(x_{h}\right)$. Considering $v_{h}\left(x_{h}\right)$ is a convex set, so the barycentric point $b\left(\left\{x_{h}(z)\right\}\right)$ of $\left\{x_{h}(z)\right\}$ can be obtained by weighted average method (taking $v_{h}\left(x_{h}\right)$ as weight). The Figure 2 describes how to generate the barycentric point of a certain total score plane by the equation (5) when there are three evaluation attributes. All the points that have been marked as stars in Figure 2 are the schemes having the same total score, and the red point which is marked as ball is barycentric coordinates point calculated by psychological weight of the evaluator.

$$
b\left(\left\{x_{h}(z)\right\}\right)=\left(\frac{\sum_{h=1}^{t} v_{1}^{h} x_{1}^{h}}{\sum_{h=1}^{t} v_{1}^{h}}, \cdots, \frac{\sum_{h=1}^{t} v_{m}^{h} x_{m}^{h}}{\sum_{h=1}^{t} v_{m}^{h}}\right)
$$




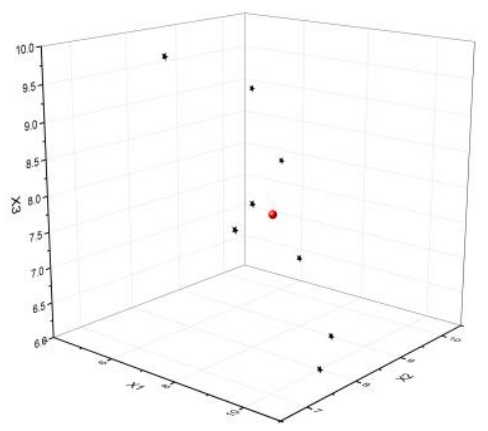

\section{Figure 2. The Generation of Barycentric Point in Three-dimensional Space}

If the training sample set $\left\{x_{k}\right\}$ is sufficient, and the training times and the scheme set $\left\{x_{h}(z)\right\}$ selected by the evaluator are also sufficient, the barycentric points $b\left(\left\{x_{h}(z)\right\}\right)$ will gradually approach the most satisfactory local solution $x^{*} \mid \mathrm{T}$ in $S_{T} \cap X$, i.e., $\lim _{h \rightarrow \infty} b\left(\left\{x_{h}(z)\right\}\right) \rightarrow x^{*} \mid \mathrm{T}$. In other words, $\lim _{h \rightarrow \infty} b\left(\left\{x_{h}(z)\right\}\right)$ is the most satisfactory local solution of evaluator $\mathrm{z}$ in $S_{T} \cap X$ (or $\Delta \mathrm{A}^{\prime} \mathrm{B}^{\prime} \mathrm{C}^{\prime}$ ). It is needed to illustrate that each time of learning can obtain the neighborhood $N\left(b\left(\left\{x_{h}(z)\right\}\right), r\left(\left\{x_{h}(z)\right\}\right)\right)$ with the center of sphere of $b\left(\left\{x_{h}(z)\right\}\right.$ and the radius of $r\left(\left\{x_{h}(z)\right\}\right)$, which is a satisfactory solution in accordance with above significance in learning for any scheme $x \in N\left(b\left(\left\{x_{h}(z)\right\}\right), r\left(\left\{x_{h}(z)\right\}\right)\right)$. With the most satisfactory solution of $z$ in $S_{T} \cap X$ or the psychological standard point $b\left(\left\{x_{h}(z)\right\} \mid \mathrm{T}\right)$, taking $b\left(\left\{x_{h}(z)\right\} \mid \mathrm{T}\right.$ as the standard, then it is not difficult for the evaluator $z$ to conduct satisfaction evaluation on all schemes $\left\{\mathrm{x}_{\mathrm{i}}=\left(\mathrm{x}_{\mathrm{i} 1}, \cdots, \mathrm{x}_{\mathrm{im}}\right) \mid\right\}$ in $S_{T} \cap X$ by local satisfaction function (3).

\subsection{The Most Satisfactory Local Solution Line}

Because $b\left(\left\{x_{h}(z)\right\}\right) \in S_{T} \cap X$ is obtained by learning, so all most satisfactory local solutions $b\left(\left\{x_{h}(z)\right\}\right) \mid \mathrm{T}[10,10 \times \mathrm{n}] \pi$ are obtained after $\mathrm{T}$ through $[10,10 \times \mathrm{n}]$. Considering that continuous change of psychological standards of the evaluator $\mathrm{z}$ on $\mathrm{T}$, bouncing changes generally don't occur, thus the change of $b\left(\left\{x_{h}(z)\right\}\right) \mid \mathrm{T}[10,10 \times \mathrm{n}]$ with respect to $\mathrm{T}$ can be regarded as continuous. In other words, the set $b\left(\left\{x_{h}(z)\right\}\right) \mid \mathrm{T}[10,10 \times \mathrm{n}]$ will be a line which is recorded as $L\left(b\left(\left\{x_{h}(z)\right\}\right)\right)$. It may be called as the most satisfactory local solution line or the psychological standard line of the evaluator. Because the scheme space $X=\left\{x_{i}=\left(x_{i 1}, \cdots, x_{i m}\right)\right\}$ is a close and tight toplogical space, thus if $\mathrm{X}$ has an infinite covering, it also can be a space to be covered by the limited covering. Therefore, subset of $\mathrm{X}$ or local satisfactory solution line of $\mathrm{z}$ $L\left(b\left(\left\{x_{h}(z)\right\}\right)\right)$ is also a space with limited covering. Therefore, $L\left(b\left(\left\{x_{h}(z)\right\}\right)\right)$ can be 
obtained by interpolation or curve fitting. The fitting algorithm of the most satisfactory local solution line $L\left(b\left(\left\{x_{h}(z)\right\}\right)\right)$ is as follows.

\subsection{Calculation of the Most Satisfactory Local Solution Line $L\left(b\left(\left\{x_{h}(z)\right\}\right)\right)$}

It is assumed that $b_{k}\left(\left\{x_{h}(z)\right\}\right) \mid \mathrm{T}_{\mathrm{k}} \in(10,10 \times m), k=1, \cdots, n$ is the most satisfactory solutions of evaluator $\mathrm{z}$ in $S_{T} \cap X$, which is calculated by the equation (5), through training or learning. Because $x^{*}=\left(10 \times \mathrm{w}_{1}, \cdots, 10 \times \mathrm{w}_{\mathrm{m}}\right)$ and $D=(10, \cdots, 10)$ are the most satisfactory solutions in $S_{10} \cap X$ and $S_{10 \times n} \cap X$, respectively, together with $\left\{b_{k}\left(\left\{x_{h}(z)\right\}\right) \mid \mathrm{T}_{\mathrm{k}} \in(10,10 \times m)\right\}$, there are $(\mathrm{n}+2)$ most satisfactory solutions in different simplex. Therefore, the polynomial equation is expressed as:

$$
G(T)=a_{10}+a_{11} T+a_{12} T_{2}+\cdots+a_{1 n+1} T_{n+1}\left(a_{j}=\left(a_{1 j}, \cdots, a_{i j}\right), i=0,1, \cdots, m\right)
$$

The most satisfactory local solution line $L\left(b\left(\left\{x_{h}(z)\right\}\right)\right)$ is fitted by interpolation formula. Here are interpolation methods of most satisfactory local solution line with only three interpolating points. Because the number of decision attributes is $m$, and the attribute values are $x_{1}, \cdots, x_{m}$, respectively, and the interpolation polynomial is set as:

$$
\begin{gathered}
G(T)=G\left(g_{1}(T), \cdots, g_{m}(T)\right) \\
\text { Where: }\left\{\begin{array}{cc}
\mathrm{g}_{1}(\mathrm{~T})=\mathrm{a}_{10}+\mathrm{a}_{11} \mathrm{~T}+\mathrm{a}_{12} \mathrm{~T}^{2} & (7-1) \\
\vdots & \\
\mathrm{g}_{\mathrm{j}}(\mathrm{T})=\mathrm{a}_{\mathrm{i} 0}+\mathrm{a}_{\mathrm{i} 1} \mathrm{~T}+\mathrm{a}_{\mathrm{i} 2} \mathrm{~T}^{2} & (7-j) \\
\vdots & (7-m) \\
\mathrm{g}_{\mathrm{m}}(\mathrm{T})=\mathrm{a}_{\mathrm{m} 0}+\mathrm{a}_{\mathrm{m} 1} \mathrm{~T}+\mathrm{a}_{\mathrm{m} 2} \mathrm{~T}^{2} & (7-m)
\end{array}\right.
\end{gathered}
$$

E.g. 1: let three most satisfactory local solutions in $S_{10} \cap X, S_{T} \cap X$ and $S_{10 \times n} \cap X$ of the evaluator $\mathrm{z}$ be:

(1) most satisfactory local solution in $S_{10} \cap X: x^{*}=\left(10 \times w_{1}, \cdots, 10 \times w_{m}\right)$

(2) most satisfactory local solution in $S_{T} \cap X$ :

$$
\left.x^{*}\right|_{\mathrm{T}}=\mathrm{b}\left(\left\{\mathrm{x}^{\mathrm{h}}(\mathrm{z})\right\}\right)=\left(\frac{\sum_{h=1}^{t} v_{1}^{h} x_{1}^{h}}{\sum_{h=1}^{t} v_{1}^{h}}, \ldots, \sum_{h=1}^{t} v_{7}^{t} v_{7}^{h} x_{7}^{h}\right) ;
$$

(3) Most ideal point, $\left.x^{*}\right|_{100 \times \mathrm{m}}=D=(10, \cdots, 10)$, three points are substituted into the following interpolation formula of Lagrange.

$g_{i}(T)=\frac{\left(T-x_{1}^{*}\right)\left(T-x_{2}^{*}\right)}{\left(x_{0}^{*}-x_{1}^{*}\right)\left(x_{0}^{*}-x_{2}^{*}\right)} a_{i 0}+\frac{\left(T-x_{0}^{*}\right)\left(T-x_{2}^{*}\right)}{\left(x_{1}^{*}-x_{0}^{*}\right)\left(x_{1}^{*}-x_{2}^{*}\right)} a_{i 1}+\frac{\left(T-x_{0}^{*}\right)\left(T-x_{1}^{*}\right)}{\left(x_{2}^{*}-x_{0}^{*}\right)\left(x_{2}^{*}-x_{1}^{*}\right)} a_{i 2}$ 
The coefficients with a number of $3 \times \mathrm{m}$ in this equation set $(7-\mathrm{j}, \mathrm{j}=1, \ldots, \mathrm{m})$ can be obtained: value of $\mathrm{a}_{\mathrm{ij}}, \mathrm{i}=1,2,3$; and equation of interpolation curve (8) of the most satisfactory solution, $\mathrm{L}(\mathrm{b}(\{\mathrm{xh}(\mathrm{z})\})$. (as z-D curve in Figure 1). Then equation set:

$$
\left\{\begin{array}{l}
\sum_{j=1}^{m} x_{i j}=T \\
\mathrm{G}(\mathrm{T})=\mathrm{G}\left(\mathrm{g}_{1}(\mathrm{~T}), \ldots, \mathrm{g}_{\mathrm{m}}(\mathrm{T})\right)
\end{array}\right.
$$

The most satisfactory local solution $\left.\mathrm{b}\left(\left\{\mathrm{x}^{\mathrm{h}}(\mathrm{z})\right\}\right)\right|_{\mathrm{T}}$ in any simplex $\mathrm{S}_{\mathrm{T}} \cap \mathrm{X}, \mathrm{T} \in[10,10 \times \mathrm{m}]$ can be obtained. If $\left.\mathrm{b}\left(\left\{\mathrm{x}^{\mathrm{h}}(\mathrm{z})\right\}\right)\right|_{\mathrm{T}}$ is exactly a practical scheme, then $\mathrm{b}(\{\mathrm{xh}(\mathrm{z})\}) \mid \mathrm{T}$ is the locally most satisfactory solution in this $\mathrm{S}_{\mathrm{T}} \cap \mathrm{X}$. Otherwise, take $\left.\mathrm{b}\left(\left\{\mathrm{x}^{\mathrm{h}}(\mathrm{z})\right\}\right)\right|_{\mathrm{T}}$ as standards, and the evaluator $\mathrm{z}$ is able to conduct satisfaction evaluation on any scheme $\mathrm{x}_{\mathrm{i}}=\left(\mathrm{x}_{\mathrm{i} 1}, \ldots, \mathrm{x}_{\mathrm{im}}\right) \in \mathrm{S}_{\mathrm{T}} \cap \mathrm{X}$ in $\mathrm{S}_{\mathrm{T}} \cap \mathrm{X}$ through (3) $\mathrm{m}$ and to find the most satisfied scheme to be the locally most satisfactory solution in $S_{\mathrm{T}} \cap \mathrm{X}$. When $\mathrm{T}$ through the interval $(10,10 \times \mathrm{m})$, most satisfactory local solution line $\mathrm{L}\left(\mathrm{b}\left(\left\{\mathrm{x}^{\mathrm{h}}(\mathrm{z})\right\}\right)\right)$ can be obtained by methods above.

\subsection{Conversion from Local to Global Satisfaction}

Generally, with the greater $\mathrm{T}$, the corresponding most satisfactory local scheme $\left(\mathrm{b}\left(\left\{\mathrm{x}^{\mathrm{h}}(\mathrm{z})\right\}\right)\right.$ of $\mathrm{T}$ in $\mathrm{L}(\mathrm{b}(\{\mathrm{xh}(\mathrm{z})\}))$ is the most satisfactory global scheme. Therefore, the globally most satisfactory solution is able to be calculated by sorting all schemes $\mathrm{x}_{\mathrm{i}} \in$

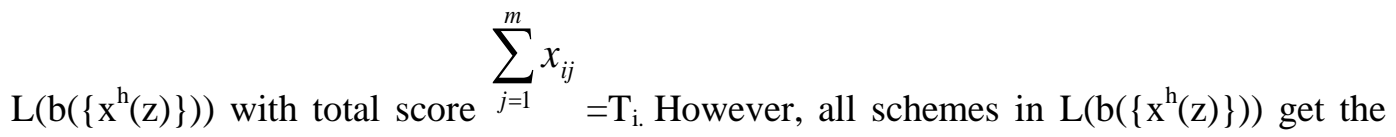
evaluation score by learning in different simplex $S_{\mathrm{T}} \cap \mathrm{X}$, therefore, this is a problem.

Because equation (3) is used to evaluate the similarity between all objects in a same simplex with $b\left(\left\{x_{h}(z)\right\}\right)$ from a standard point $\left.\mathrm{b}_{\mathrm{i}}\left(\left\{\mathrm{x}^{\mathrm{h}}(\mathrm{z})\right\}\right)\right|_{\mathrm{Ti} \in(10,10 \times \mathrm{m})}$, and it's not for the globally similarity, so in order to get the globally most satisfactory scheme, we need to present an evaluation equation that is able to evaluate all schemes of $\mathrm{L}(\mathrm{b}(\{\mathrm{xh}(\mathrm{z})\}))$ globally .

After adjustment, we find that it only needs to let $\lambda(x, z)$ of (2) be:

$\lambda(x, z)=\left(\frac{\sum_{i=1}^{m} x_{i j}}{\sum_{j=1}^{m} X_{j}}\right)^{S}$

Where $\sum_{j=1}^{m} X_{j}$ is the sum of $\mathrm{Xj}$ which is with each attribute to be full marks, and $\sum_{i j=1}^{m} x_{i j}$ is sum of scheme $\mathrm{x}_{\mathrm{ij}}, \mathrm{S}=\left(\frac{\sum_{j=1}^{m} X_{j}}{\sum_{i=1}^{m} x_{i j}}\right)$. Substitute into (3), and obtain: 
$\operatorname{sat}(\mathrm{x}, \mathrm{z})=\left(\frac{\sum_{\mathrm{i}=1}^{\mathrm{m}} \mathrm{x}_{\mathrm{ij}}}{\sum_{\mathrm{j}=1}^{\mathrm{m}} \mathrm{X}_{\mathrm{j}}}\right)_{*}^{\mathrm{S}} \exp \left(-\frac{\sum_{j=1}^{m} w_{j} \mid x_{j}-b\left(x^{h}\left(z_{j}\right) \mid\right.}{\sum_{j=1}^{m} w_{j}\left(b\left(x^{h}\left(z_{j}\right)-\delta_{j}\right)\right.}\right)$

It is able to make the satisfaction $\operatorname{sat}\left(\mathrm{x}_{\mathrm{i}}, \mathrm{z}\right)$ be basically accordant in the whole scheme space by equation (11), thus $\lambda(\mathrm{x}, \mathrm{z})$ is called as the coefficient of overall concordance.

According to equation (11), the base number reflects the total points $\sum_{i j=1}^{m} x_{i j}$ of each attribute value of the scheme, and proportion in full marks $\sum_{j=1}^{m} X_{j} o$.

\section{Problems of Current ACEM}

Although ACEM has resolved many practical problems, there is a problem, i.e., a few unreasonable results will appear during the conversion from local to global satisfaction. Although some scholars have tried to solve this problem from various ways [16-18], there is no good method to solve it completely. Here is detailed analysis on this problem with college entrance examination scores of a Shandong senior high school as data source.

E.g.2 The data source includes 4 attributes and 2,500 evaluation objects. Firstly, select two planes of total score (hereinafter referred to as PTP), PTP560 and PTP533, and the evaluator marks all sample points in these two planes. By equation (5), barycentric coordinates of these two planes can be obtained as 215.1667, 115.7083, 113.4167 and 115.7083 for plane 1 , and $194.8059,111.8498,110.7692$ and 115.5751 for plane 2 . Then obtain barycentric curves of four attributes respectively by Formula (9), and they are blue curves in Figure 3. The red straight lines assume that preference of the evaluator does not change with the change of total score, in other words, the weights are fixed. Conduct evaluation on sample points of the plane with total score of 549 by equation (3), and the evaluation results is shown as Table 2. According to analysis the results, the weight of index $\mathrm{x} 4$ is the biggest, meanwhile, it also considered the equilibrium of each attribute. In conclusion, evaluation result of ACEM on local satisfaction is very reasonable.

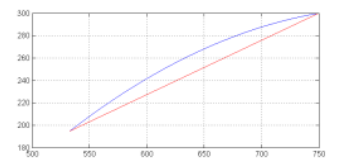

Attribute X1

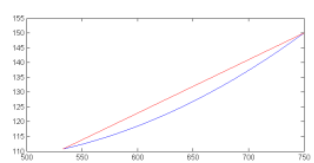

Attribute X3

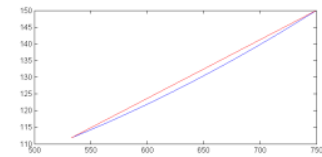

Attribute X2

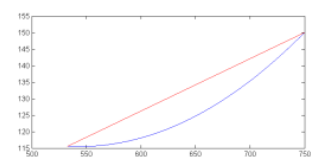

Attribute $\mathrm{x} 4$

Figure 3. The Barycentric Curves of Various Attributes and The Straight Line with a Fixed Weight. 


\section{Table 2. The Evaluation Results of Local Satisfactory Degree with Total Score Plane of 549.}

\begin{tabular}{|c|c|c|c|c|c|}
\hline ID & $\mathbf{X 1}$ & $\mathbf{X 2}$ & $\mathbf{X 3}$ & $\mathbf{X 4}$ & Score \\
\hline 重心坐标 & 207.1210 & 114.1059 & 112.2614 & 115.5117 & \\
\hline $\mathbf{1}$ & 216 & 117 & 102 & 114 & 0.8399 \\
\hline $\mathbf{2}$ & 204 & 111 & 111 & 123 & 0.8961 \\
\hline $\mathbf{3}$ & 212 & 101 & 115 & 121 & 0.8262 \\
\hline $\mathbf{4}$ & 201 & 107 & 132 & 109 & 0.7520 \\
\hline $\mathbf{5}$ & 211 & 126 & 101 & 111 & 0.8043 \\
\hline $\mathbf{6}$ & 218 & 113 & 111 & 107 & 0.8404 \\
\hline $\mathbf{7}$ & 179 & 126 & 113 & 131 & 0.6518 \\
\hline
\end{tabular}

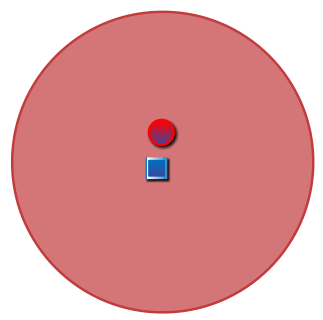

539

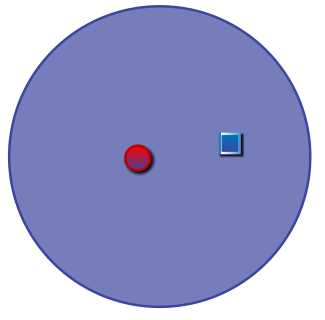

586

Figure 4 local satisfaction comparison between two total score planes

After global satisfaction has been converted from local satisfaction by (10), most of the data is relatively reasonable. However, there is a small part of unreasonable data, and the main reason is the error from conversion equation (10). As shown in Figure 4, in PTP539 and PTP586, red points are barycentric coordinates and blue points are evaluation objects. Obviously, points of local satisfaction in PTP539 are higher than points in PTP586, because it is more close to the barycentric coordinates of its plane. However, in terms of global satisfaction, the points in PTP586 should be higher than points in PTP539. There are two ideas for solving this problem. First is the re-fitting the conversion function; [15] has tried to solve the problem in this way, but it only solved specific problems. It couldn't eliminate the error completely. The second idea is to abandon local satisfaction, and to directly calculate global satisfaction to avoid errors caused by conversion from local to global satisfaction, which is the solution of this paper.

\section{Solution of Global Satisfaction}

The core of ACEM is the dynamic weight, i.e., psychological weight of evaluator will dynamically change with the change of total score of evaluation objects. Therefore, as long as we can calculate different psychological weights of the evaluator for each PTP, and take these weights in evaluation objectives, then evaluation is able to be conducted directly from an global perspective, and dynamic weight of the evaluator is able to be reflected. In this way, global satisfaction can be directly calculated, thus errors caused by conversion from local to global satisfaction can also be avoided. The equation (12) is the psychological barycentric curve corresponding to the evaluator for an index. e.g., the curve in Figure 5, horizontal ordinate $\mathrm{T}$ is total score and vertical coordinate ${ }^{x_{1}}$ is point value of an attribute. Formula (13) assumes that the preference of the evaluator for this attribute is not dynamically changed, thus it is a straight line, i.e., the weight is constant, e.g., the straight line in Figure 5. When " $\mathrm{t}$ " is same, and difference between ${ }^{g_{1}}$ of (12) and $l_{1}$ of (13) is up to max, the corresponding point is the turning point of $g_{1}-l_{1}$ of the 
equation. The turning point means the psychological weight of the evaluator for certain evaluation attribute has been offset to the max when changing according to the total score.

$\mathrm{g}_{1}(\mathrm{~T})=\mathrm{a}_{10} \mathrm{~T}^{2}+\mathrm{a}_{11} \mathrm{~T}+\mathrm{a}_{12}$

$l_{1}(T)=b_{10} T+b_{11}$

Equation (14) provides the way to calculate the max offset. By equation (15), coordinates of turning point $T_{p}$ can be obtained, as well as the max offset distance $d_{1}$, e.g., the straight line between ${ }^{g_{1}}$ to $l_{1}$ in Figure 5 . From the global perspective, because ${ }^{g_{1}}$ is below $l_{1}$, thus corresponding $x_{1}^{p}$ of $T_{p}$ should be the point has the lowest weight of attribute ${ }^{x_{1}}$. If $g_{1}$ is above $l_{1}, x_{1}^{p}$ should be the point has the highest weight of attribute $^{x_{1}}$.

$d_{1}=\operatorname{argmax}_{\mathrm{T} \in[1, \mathrm{t} 3]}\left|\mathrm{g}_{1}(T)-\mathrm{l}_{1}(T)\right|$

$\frac{d\left(g_{1}-l_{1}\right)}{d T}=0 \Rightarrow 2 a_{10} T+a_{11}-b_{10}=0 \Rightarrow T=-\left(a_{11}-b_{10}\right) / 2 a_{10}$

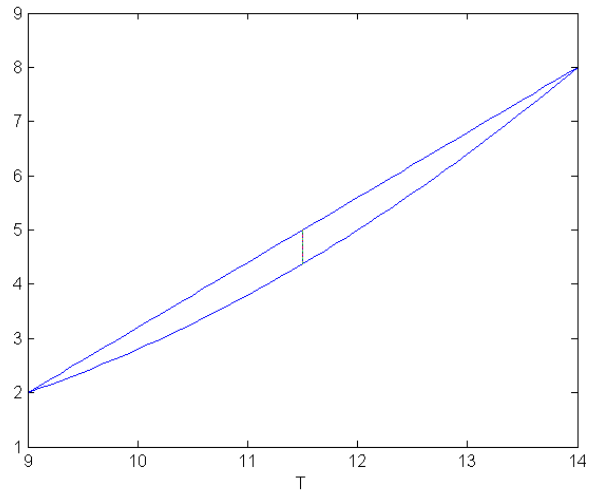

Figure 5. The Offset Distance of Maximum Weight

It is assumed that weight interval of attribute $x_{1}$ is within $[\alpha, 1]$, and then corresponding barycentric coordinates points of ${ }^{x_{1}}$ in each PTP can be obtained by Formula (16). In the same way, dynamic weights of other attribute are also obtained. The final satisfaction function is shown as Formula (17).

$$
\begin{aligned}
& w_{1}=\alpha+\left(\left|\mathrm{g}_{1}(T)-\mathrm{l}_{1}(T)\right| / d_{1}\right) *(1-\alpha) \\
& \operatorname{sat}\left(x_{i}\right)=\left(\frac{\sum_{j=1}^{m} w_{j} x_{i j}}{\sum_{j=1}^{m} w_{j}}-\underset{x \in[1, \mathrm{n}]}{\arg \min } \frac{\sum_{j=1}^{m} w_{j} x_{i j}}{\sum_{j=1}^{m} w_{j}}\right) /\left(\underset{x \in[1, \mathrm{n}]}{\arg \min } \frac{\sum_{j=1}^{m} w_{j} x_{i j}}{\sum_{j=1}^{m} w_{j}}-\underset{x \in[1, \mathrm{n}]}{\arg \max } \frac{\sum_{j=1}^{m} w_{j} x_{i j}}{\sum_{j=1}^{m} w_{j}}\right)
\end{aligned}
$$


Author names and affiliations are to be centered beneath the title and printed in Times New Roman 12-point, non-boldface type. Multiple authors may be shown in a two or three-column format, with their affiliations below their respective names. Affiliations are centered below each author name, italicized, not bold. Include e-mail addresses if possible. Follow the author information by two blank lines before main text.

\section{Evaluation Principles and Simulation Experiment of IACEM}

To analyze if the final evaluation result getting by ACEM is reasonable or not, a principle is needed. The advantage of ACEM is to adopt the weight priority principle for evaluated objects in the same level of total score, and to use total score priority principle for evaluated objects in different levels of total score. This paper uses these features as an evaluation principle for ACEM, concretely speaking, for example, in E.g.2, it is assumed that 560-562 belongs to the same level of total points; the weight of $\mathrm{x} 1$ is higher than other attributes through calculating the psychological weight of the evaluator. Total score of an evaluated object are 560, and score of attribute $\mathrm{x} 1$ is 216 ; total score of another evaluated object are 562, but the score of attribute $\mathrm{x} 1$ is only 200 . If the final evaluation result is that the score of the first evaluated object is higher than the second, the result is reasonable. Because in the same level of total score weight priority is preferential. But if the total score of the second evaluated object is 563 , the score of attribute $\mathrm{x} 1$ is also only 200. Because 563 and 560 are not in the same evaluation level, the reasonable result should be the final score of the second evaluated object is higher than that the first one.

Based on the above assessment principle, the imporved model is used to evaluate the data in E.g.2 again, and the results getting from ACEM has been analyzed. Figure 6 shows how many ranking results in different total score intervals with dynamic weight have been changed when compared to those without dynamic weight. The changing trend of evaluation result is similar to the weight curve, and this means in center part of PTP, changing range of weight is bigger or smaller than two ends. Thus the influence on the final evaluation result will be greater.

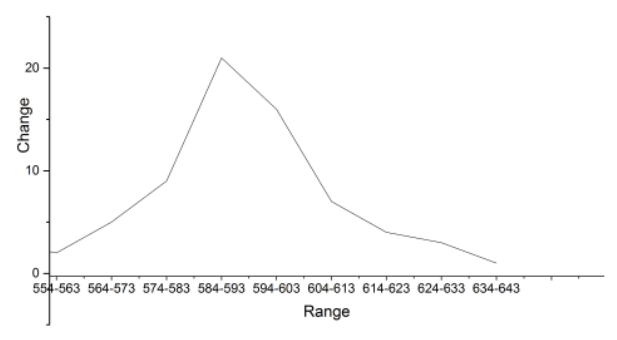

Figure 6. Evaluation Result Change Situation in Different Interval of Total Score

It is assumed that the range of a same PTP is 2. In this case, if a total score of the evaluated object is lower 3 than that of another object, but gets higher final evalution result, this situation is regarded as unreasonable, because it is not in accordance with total score priority principle. Figure 7 shows the error of results getting by ACEM under this principle. In ACEM it is not able to solve the problem even though we know the existence of error. 


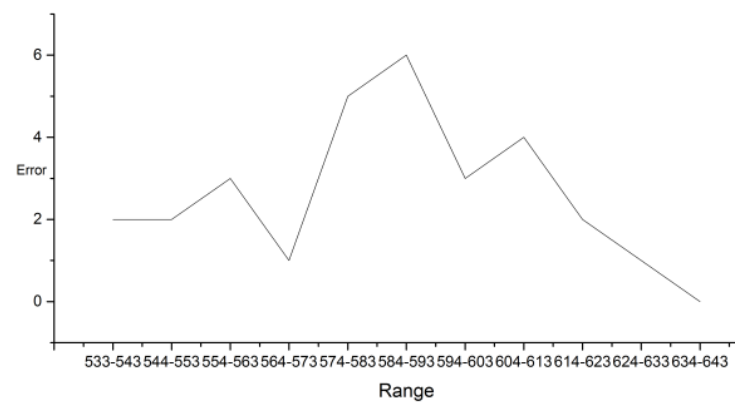

Figure 7. Evalution Change Analysis on Inconformity with the Total Score Priority in ACEM

The IACEM refelcts the weight priority principle in a same PTP and the total score priority principle in deferent PTP as well as refelcts the dynamic weight of the evaluator. During the practical evaluation, the setting of same PTP can be different according to different specific situations. In E.g 2, the same total score can be set as 2 or 10. If we abtain the reasonable results form a same evaluation interval of 10 , then it may be unreasonable from a same evaluation interval of 2 . In this case, the reasonable evaluation result is able to be achieved by adjusting the weight range $\alpha$. Lessen the $\alpha$ to amplify the impact of dynamic psychological weight of the evaluator; it can be applied to a PTP with a large range. Enlarge the $\alpha$ to narrow the impact of dynamic psychological weight of the evaluator; it can be applied to a PTP with a small range. Assuming that it is enlarged to 1 , that indicates the psychological weight of the evaluator becomes a stright line. E.g. In Figure 8, when PTP is 2, changing degree of ranking is able to be changed by adjusting the $\alpha$. When $\alpha \geq 0.95$, ranking result is completely in accordance with two principles of the assessment. At this moment, the assessment model is reasonable and correct.

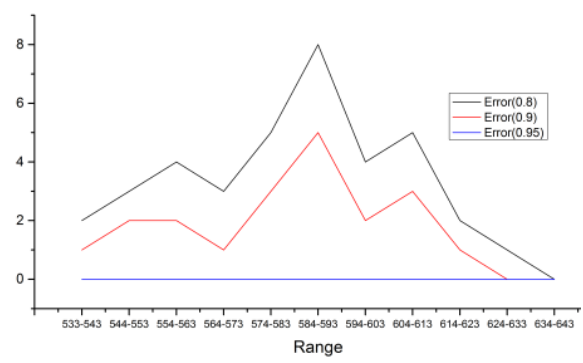

Figure 8. The Effects of Changing $\alpha$ on the Ranking Result

\section{Conclusions}

This paper analyzes the main problems encountered by the ACEM, and puts forward the corresponding improved evaluation model. The improved model can calculate the global satisfaction, so as to avoid the conversion errors from the local satisfaction to the global satisfaction. This paper also proposes the evaluation principles and specific methods as well as gives the concrete method of the priority principle of satisfactory total score by adjusting the lower limit of the weight range. Finally, the proposed simulation experiment verifies the correctness and rationality of the improved model. 


\section{References}

[1] T. L. Saaty, The Analytic Hierarchy Process: Planning, Priority Setting, Resource Allocation, McGrawHill(1980).

[2] T. L. Saaty, An exposition of the AHP in reply to the paper "remarks on the analytic hierarchy process", Management Science, v.36 n.3, p.259-268, March(1990).

[3] T. L. Saaty, Decision Making with Dependence and Feedback: The Analytic Network Process(1996).

[4] T. L. Saaty, The analytic hierarchy and analytic network measurement processes: Applications to decisions under Risk, European Journal of Pure and Applied Mathematics, Vol.1, No.1, pp. 122-196, (2008).

[5] C. G. E. Boender , J. G. de Graan, F. A. Lootsma, Multicriteria decision analysis with fuzzy pairwise comparisons, Fuzzy Sets and Systems, v.29 n.2, p.133-143, January(1989).

[6] Duan Xueyan, Liu Yongchang,Evaluation on 3PL's core competence based on method of attribute theory,Journal of Shanghai Maritime University, Vol. 27(1)(2006).

[7] Wu Qinfan, Feng Jiali,A Kind of Evaluation and Decision Model Based on Analysis and Learning of Attribute Coordinate. Journal of Nanjing University(Natural Science), Vol. 39(2) (2006).

[8] Feng Jiali, Zhao TangA, KDD Model Based on Conversion of Quantity-Quality Features of Attributes, Journal of Computer Research and Development,Vol. 37(9)(2000).

[9] Feng Jiali, Feng Jingjuan, Granularity transformation of qualitative criterion,orthogonality of qualitative mapping system,and pattern recognition. IEEE International Conference on Granular Computing (2006), pp. 211-216.

[10] Duan Xueyan, Yu Siqin,Xu Guanglin,Feng Jiali,Evaluation on Comprehensive Competitiveness of Inland Port Based on The Method of Arrtribute Theory,Journal of Pattern Recognition \& Image Proressing.Vol.(4),pp.373-380(2013).

[11] Xu Guanglin, Wang Lifang,Evaluation of Aberrant Methylation Gene Forecasting Tumor Risk Value in Attribute Theory, Journal of Basic Science and Engineering,Vol.16(2) (2008).

[12] Feng Jiali, Bi Jing-ying,Lung Cancer Cells' Recognition Based on Attribute Theory, Journal of Guangxi Normal University(Natural Science Edition), Vol.29(3)(2011).

[13] $\mathrm{Xu}$, Guanglin, Min Song,Research on Multi-agent Comprehensive Evaluation Model based on Attribute Coordinate, IEEE International Conference on Granular Computing (GrC),pp. 556 - 562(2012).

[14] Li Jianli, Liu Yongchang,Comprehensive Assessment \& Optimized Selection of Supplier in SCM Based on Attribute Theory,Logistics Technology,Vol.26(5)(2007).

[15] Duan, Xueyan, Guanglin Xu, Siqin Yu,Application of attribute theory for container throughput forecast, IEEE International Conference on Granular Computing $(\mathrm{GrC})(\mathbf{2 0 1 2})$.

[16] Xu Guanglin, Liu Yongchang,Evaluation of Chinese Software Enterprises' Core Competence in Attribute Theory,Journal of Guangxi Normal University (Natural Science Edition) (2006), Vol. 24(4).

[17] Xu Xiaolin, Xu Guanglin,Feng Jiali,A kind of synthetic evaluation method based on the attribute computing network, IEEE International Conference on Granular Computing (GrC)(2009).

[18] Xu Guanglin,Feng Jiali, Liu Yongchang, Pattern Recognition Method Based on the Attribute Computing Network, Journal of Computer Research and Development (2008), S1.

\section{Authors}
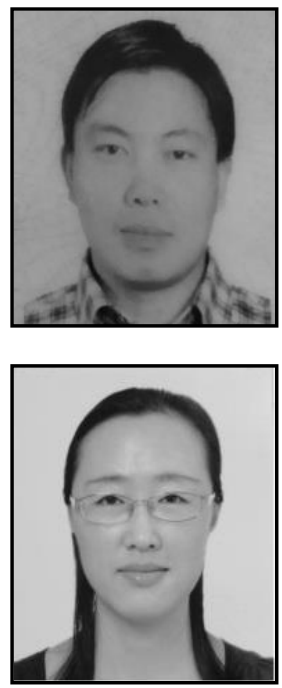

Xu Guanglin, he obtained his doctorate from the shanghai maritime university in 2008. His research focuses on Attribute network, Machine learning, Pattern Recognition, and Decision Making.

Xiaolin Xu, received the M.S. degree from Shanghai Maritime University respectively in 2003, in computer application technology. She works currently as an associate professor in Shanghai Second Polytechnic University. Her research interests include collective intelligence, machine learning, multi-index decision analysis and evaluation. 
International Journal of Grid and Distributed Computing

Vol. 9, No. 9 (2016) 